\title{
Task Allocation and Scalability Evaluation for Real-Time Multimedia Processing in a Cluster Environment
}

\author{
Jerzy Proficz ${ }^{(\varpi)}$ and Henryk Krawczyk ${ }^{(\varpi)}$ \\ Gdansk University of Technology, Gdansk, Poland \\ jerp@task.gda.pl, hkrawk@eti.pg.gda.pl
}

\begin{abstract}
An allocation algorithm for stream processing tasks is proposed (Modified Best Fit Descendent, MBFD). A comparison with another solution (BFD) is provided. Tests of the algorithms in an HPC environment are described and the results are presented. A proper scalability metric is proposed and used for the evaluation of the allocation algorithm.
\end{abstract}

Keywords: Scalability $\cdot$ Task allocation $\cdot$ HPC $\cdot$ KASKADA platform

\section{Introduction}

The pressure of the society demanding secure public environment enforced the governments and local authorities to deploy special measurements related to monitoring of cities and the surrounding areas. The ubiquitous recording devices, installed in the public space, enable recording and post mortem analysis of dangerous events. Currently, however, the main goal is to perform such analysis in real-time, mainly to prevent the dangerous situations from happening at all.

The Mayday Euro 2012 project was conceived to provide the proper means for introducing the online multimedia stream processing services into the world of supercomputer datacenters. KASKADA platform [4], an HPC system realizing the above objectives, including execution of soft real-time multimedia applications and underlying services, was developed at the TASK Academic Computer Center of the Gdansk University of Technology in Poland. The platform was deployed on a computer cluster Galera, consisting of 672 compute nodes and 4032 processor cores.

KASKADA platform supports two main groups of functionalities, the first one is related to development of multimedia applications and services, while the second one enables their execution in the supercomputer environment. In this article we focus on

The experiments described in this article were performed at the Academic Computer Center (TASK) of Gdansk University of Technology. The work was realized as a part of MAYDAY EURO 2012 and CD NIWA projects, Operational Programme Innovative Economy 2007-2013, Priority 2 "Infrastructure area R\&D". 
the latter, specifically on task management issues related to resource allocation and their influence on the system scalability. We propose a new heuristic approach supporting task allocation on a compute cluster with the soft real-time constraints.

Our contribution in this paper is as follows:

- a new allocation algorithm for real-time multimedia tasks specifically tailored for non-linear resource increase,

- a scalability metric adopted for real-time multimedia processing,

- an empirical evaluation of the proposed algorithm in a real computer cluster environment, showing its superior scalability in comparison with the typical approach.

The following section provides background information about the described problem, including main definitions and related works. The next section is related to the scalability concept and its meaning for real-time multimedia stream processing systems. Then we present the proposed allocation algorithm and its evaluation in a real data center environment. Finally, we provide conclusions and plans for future works.

\section{Problem Description and Related Works}

KASKADA platform provides facilities for acquisition, archiving and processing of large numbers of multimedia streams, recorded by different types of devices, including video cameras (PAL and HD), thermographic (infrared) cameras, and microphones. Such massive data flow requires well established network connections: an external fiber network, used for connecting the recording devices, as well as interconnect one, used for the exchange of partially processed (intermediate) data. Moreover, this flowing data needs to be processed by tasks realizing complex algorithms, which usually requires a high performance computer [4].

During our earlier research, we observed a certain phenomenon related to the allocation of more than one multimedia stream processing task on a single compute node $c$. In such conditions, the total processor utilization of a set of tasks $T$ increases more than the sum of each task processor utilization $\left(\gamma_{i}\right)$, i.e.:

$$
\gamma(c, T) \geq \sum_{i=1}^{|T|} \gamma_{i}
$$

Moreover, in [7] we proposed a method for estimating the total utilization for a given set of tasks $T$ and a specific node $c$. We proposed the following formula:

$$
\gamma(c, T) \approx \eta_{h}\left(\sum_{i=1}^{|T|}\left|S I_{i}\right|\right) \sum_{i=1}^{|T|} \gamma_{i}
$$

where $\eta_{h}$ is a correction function determined experimentally for a specific type of the processed stream $h$, and $S I_{i}$ is a set of input streams for task $i$.

The typical process of service execution in KASKADA platform is performed as follows: the user using his/her workstation invokes a feature in the web application, which forwards this request trough the web service interface. The parallel processing management server detects the type of the call, and if a complex services is involved, it 
decomposes the complex service structure into simpler ones, then selects the proper tasks and finally performs an allocation of the resources in the cluster. Finally, the threads and processes of the tasks are started and the initial notification delivered to the client. Afterwards, during the task execution, the procedure of monitoring is performed and the results (output multimedia streams and event messages) are delivered and archived.

A resource allocation algorithm accepts a set of tasks to be allocated as the input and assigns these tasks to the cluster nodes. The algorithm considers two constraints. The former is related to the real-time nature of the allocated tasks, the chosen nodes need to perform proper provisioning by providing enough computing resources, in our case the processor time. The latter constraint, which is not obligatory, is related to general cluster management, specifically deciding if the cluster is to be loaded uniformly or should the tasks be concentrated on a minimal number of nodes. Because of the possibility of blocking new computationally-intensive tasks when all nodes are partially utilized, as well as the opportunity for utilizing power saving strategies, we decided to use the concentration approach.

In the related works, the scalability of a real-time system was considered in [1], where a set of five scheduling algorithms was implemented in LITMUS (LInux Testbed for MUltiprocessor Scheduling in Real-Time systems) environment and used for testing a Sun Niagara multicore platform. The scalability was analyzed for a fixed number of logical processing cores (32) in comparison to the number of executed tasks. The results were visualized as schedulability, the factor measuring ratio of the non-delayed computations as a function the utilization cap, i.e. the load of the tested processors.

Another example of scalability assessment can be found in [2]. It describes a realtime cyberinfrastructure, including a hardware and software platform for Real-time Online Interactive Applications (ROIA) - mainly Massively Multiplayer Online Games (MMOG). They assume the platform is scalable when it is able to maintain the real-time constraints as the number of users increases, by using parallel and distributed mechanisms in application design while increasing the server number. The results were presented as the average CPU utilization for a given server number, in opposition to the served clients number.

In [6] Yu Tang presented the Pull-Based Distributed Scheduling (PBDS) algorithm for a cluster architecture of real-time servers. The described scalability tests were performed for the number of cluster nodes increasing from 10 to 30, and two load levels: light and heavy, with varying transfer rates expressed in $\mathrm{Kb} / \mathrm{s}$. The results were presented as the average response time of the system (in seconds) and the overall throughput (in $\mathrm{Kb} / \mathrm{s}$ ) in comparison to the number of nodes, separately for the light and heavy loads.

The common characteristic of the described scalability evaluations is the usage of some performance parameter like response time, throughput or generated frames per second, as a speedup substitute. Then, like in the formal definition [5], these measurements are presented as a function of either the computation units number (e.g. nodes, GPUs) or the problem size (utilization cap or data size in the definition [5]). The scalability itself is not directly evaluated, except from the approximating statements like it "is nearly linear" in [2]. 


\section{Scalability Definition}

Scalability is intuitively defined as a capability to hold performance up across machine sizes and problem sizes. This definition can be formally formulated using the concept of speedup, which is a two-dimensional function of a given system [5]:

$$
\varepsilon(p, n)=\frac{\tau(1, n)}{\tau(p, n)}
$$

where $p$ is the number of the installed computation units (e.g. processors, cluster nodes), $n$ is the input data size, and $\tau(p, n)$ is the measured time of computation for the given computation units number and data size. For a fixed data size, the ideally scalable system speedup is defined as $S p(p)=p$, the high performance scalability assumes $p /$ $2<S p(p)<p$ for a given $p$ range. Similarly, for a fixed computation unit size, $p=P$ and a given data size range, the system highly scalable when $P / 2<S p(n)<P$. Kuck in [5] defines other ranges of speedup for intermediate cases, as well as unacceptable scalability levels.

KASKADA platform is a system realizing computations on continuously flowing data - streams. The processing is performed in real-time, with possible delays, and even with acceptable low level data loss. However, the computation times are fixed and they are not dependent on the data size (e.g. video resolution or fps) nor the number of computation units (nodes); otherwise the results returned to the user would be unacceptable. Thus the formula (3) cannot be used directly, because the speedup would always equal $1(\varepsilon(p, n) \approx 1)$.

The more appropriate scalability metrics, concerning quality of processing, was proposed by Jogalekar in [3]. It is based on the productivity defined as:

$$
\iota(p)=\frac{\lambda(p) f(p)}{\kappa(p)}
$$

where $p$ is the scale of the system (e.g. the number of installed computation units, processors, nodes etc.), $f(p)$ is the average value of each response, directly related to the quality provided by the system, and $\lambda(p)$ is the throughput of the system, $\kappa(p)$ is the cost of the system. The scalability metric itself is defined as the ratio of the productivity of two scales: $p_{1}$ and $p_{2}$ :

$$
\psi\left(p_{1}, p_{2}\right)=\frac{l\left(p_{2}\right)}{l\left(p_{1}\right)}
$$

Usually the scale $p_{1}$ is fixed, so the above metric can be written as $\psi\left(p_{2}\right)$. In the case of KASKADA platform, we decided to use normalized output data loss as a quality metric:

$$
f(p)=\frac{1}{1+\frac{\varphi(p)}{L}}
$$


where $\varphi(p)$ is the measured fraction of output data lost and $L$ is the acceptable level of data loss. We arbitrary set its value to $1 \%$, thus the above equation can be simplified as:

$$
f(p)=\frac{1}{1+100 \varphi(p)}
$$

For the platform at a given scale $p$, we can measure the throughput $\lambda(p)$ as a number of video frames (or other stream elements) entering the system, which is directly proportional to the number of processed streams. On the other hand, we assume the cost of the system is directly proportional to the number of used nodes, thus we can denote $\kappa(p)$ as the number of used nodes. However, the allocation algorithm can also allocate a node only partially, thus this value can be fractional. Moreover, we also assume the value for fixed referential productivity $l\left(p_{1}\right)$ is to be measured for a minimum scale (number of used nodes), but with the data loss being below the threshold $\varphi(p)<L$.

The above scalability metric has a specific feature: its values can be greater than 1.0. It is caused by the granularity of the performed services and discretion of a single node, e.g. for one node the system can allocate only one service, however for two nodes it can provide resources for 3 services, due to the possibility of allocating the tasks of the third service on both nodes.

\section{Allocation Algorithm}

We can assume two main allocation strategies: load balancing and load concentration. The former is widely used for uniform workload distribution, especially when the goal is the maximum resource usage and the computations scale well. The latter causes the resources to be used partially, which can be important for energy savings when the unused hardware (e.g. compute nodes) can be switched off.

One of the most important elements of computation management is proper task provisioning. This is a critical issue for real-time tasks, when resource starvation can cause indivertible loss of processed data and unacceptable decrease of reliability. Thus a large pool of unused resources is necessary for execution of large number of such realtime tasks, which makes the load concentration strategy more appropriate. Moreover, in our case the fact that two or more cooperating tasks are located on the same compute node can be used for network traffic minimization, as well as for decreasing the processor and memory load in the case when data encoding and packaging can be skipped.

In the case of KASKADA platform, load concentration can be realized using an allocation algorithm resolving the well known bin packing problem with variable size of the bins. We assume the task (processor) load is the size of the packed element, and the free resources - (partially) unused computation nodes are the bins. The maximum additional loads which can be placed on the nodes correspond to the capacity of the bins. The above problem is NP-hard, we proposed and analyzed a set of heuristics in [8], where the simulation showed that for the given environment BFD (Best Fit Descending) algorithm is the most suitable.

KASKADA platform is responsible for proper allocation and provisioning of multimedia processing tasks, executed with the soft real-time constraints. We denote task $i$ as: 


$$
t_{i}=\left(a_{i}, S I_{i}, S O_{i}, \gamma_{i}\right)
$$

where $a_{i}$ is the task algorithm, $S I_{i}$ is the set of input streams, $S O_{i}$ is the set of output streams, and finally $\gamma_{i}$ is processor utilization generated by the task in the case it is executed on a node exclusively. Because of the computation specifics, we assume the memory and network bandwidth are not a bottle-neck, thus they can be properly provisioned.

KASKADA platform realizes a service call by executing a set of interconnected computation tasks. If the allocation algorithm allocates more than one task to a specific node, its resources, especially processor cores and memory bus must be shared. Because of the specific type of computation, i.e. multimedia processing, a nonlinear processor load increase occurs [7], namely a task executed exclusively on a node causes lower processor utilization than the same task executed together with other tasks.

To provide the proper allocation, i.e. without the possibility of task starvation, the allocation algorithm requires a prediction function for processor utilization, which should accept the task descriptions (denoted in Eq. 1) as input, and provide as a result the total processor utilization of all tasks executed together on a node. We assume the function is non-decreasing with the increasing task set. We proposed the realization of such a function in [7], it consists of a product of two components:

$$
\gamma(c, T) \approx\left(\sum_{i=1}^{|T|} \gamma_{i}\right) \cdot \eta_{h}\left(\sum_{i=1}^{|T|}\left|S I_{i}\right|\right)
$$

the former is a plain sum of all processor utilizations caused by the tasks (the set $T$ ) executed on a node exclusively, and the latter is the correction function $\eta_{h}$, determined experimentally for a specific media type $h$. The above method is realized in the PRED function in listing in Fig. 1. The linear utilization util is computed in the loop (lines 5-7) and the correction function $\eta_{\text {pal }}($ str) (used in line 8) is kept as an array in memory. The function $\omega(c)$ returns the array of the tasks already allocated to the node $c$, $|t . S I|$ and $t . \gamma$ are respectively the number of input streams and the initial utilization (measured on a node exclusively) of the task $t$.

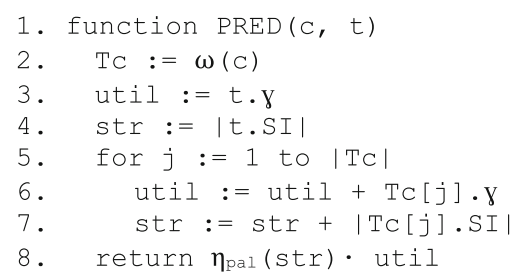

Fig. 1. The prediction function formula based on the correction function (2) from [7]

The listing in Fig. 2 presents the pseudo-code of the proposed allocation algorithm MBFD (Modified Best Fit Descending). The provided set $T$, consisting of tasks to be allocated on the nodes of the cluster $C$, is used to create the list $\mathrm{T}^{*}$, ordered descendingly by the task processor utilizations $\left(\gamma_{i}\right)$. The algorithm divides the cluster (cases in lines 4,16 and 24 ) to be used by three types of the multimedia streams: video PAL (set $\mathrm{C}_{\mathrm{PAL}}$ ), 
video $\mathrm{HD}$ ( $s e t \mathrm{C}_{\mathrm{HD}}$ ), audio/non-multimedia (set $\mathrm{C}_{\mathrm{NIL}}$ ), with possible extension for additional types implemented in the function $\nu(t)$, which detects the stream type of the task $t$ (line 3). Because of the high non-linearity of tasks processing HD streams, only one such task can be executed on a single node.

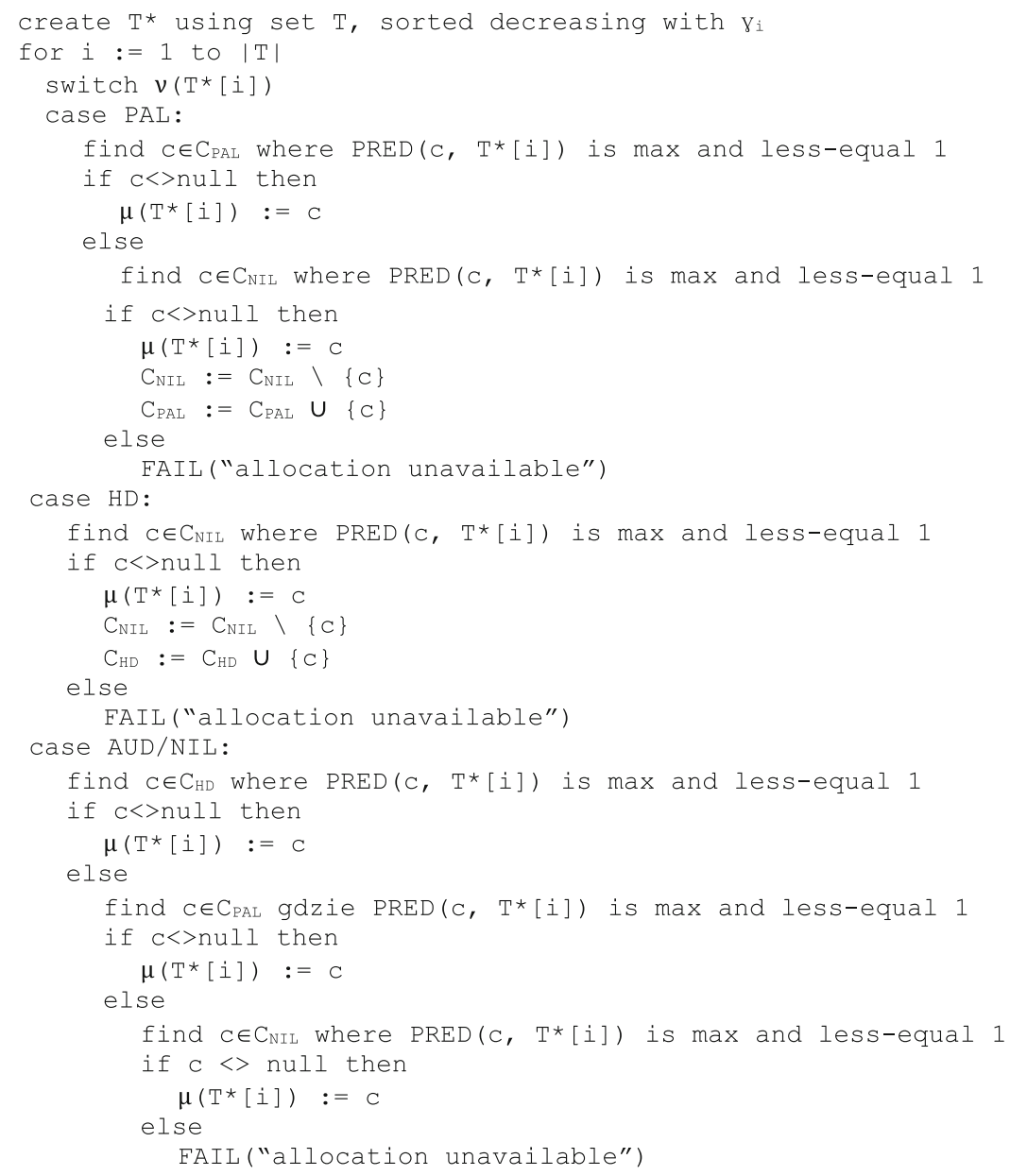

Fig. 2. The allocation algorithm MBFD: Modified Best Fit Descending

The main loop of the algorithm iterates trough the list of tasks $\left(\mathrm{T}^{*}\right)$. Every consecutive task is matched to the to the best node, i.e. the one which utilization, after the task allocation, will be maximal but not greater than 1 . The low influence of tasks with audio or without streams implies that the algorithm can group them with both HD and PAL streams (lines 25, 29, 33), however tasks processing PAL and HD streams should not be grouped together on the same node, thus they can use the audio/no stream (from set $\mathrm{C}_{\mathrm{NIL}}$ ) and free nodes (lines 5, 9 for PAL and line 17 for HD). In case there are no matching nodes in the cluster for the task being allocated, an error (failure) is reported and the 
algorithm is finished. The outcome allocation is stored as an associative array $\mu(t)$, with the key being the allocated task $t$, and the value the best matching node of the cluster $c$.

\section{Experimental Evaluation}

The experiments were performed using a Galera computer cluster, placed at the TASK Academic Computer Center of the Gdansk University of Technology. For the algorithm evaluation we used an increasing set of compute nodes, starting with one and up to one hundred. Each node consists of two Intel Xeon E5345 $2.33 \mathrm{GHz}$ (8 cores) processors, 8 GB RAM, and 20 Gbps Infiniband network interface connected in the fat tree structure. The evaluation was performed against four benchmark services. All of them processed PAL video streams, and consist of simple services executed as connected computation tasks. Table 1 presents the description of the services. The motivation to select these services was to examine the most popular algorithms used by KASKADA platform users, as well as to check the allocation algorithm against various workloads.

Table 1. The benchmark services

\begin{tabular}{|c|c|c|c|c|c|}
\hline ID & Functionality & $\begin{array}{l}\text { Input } \\
\text { stream no }\end{array}$ & $\begin{array}{l}\text { Output } \\
\text { stream } \\
\text { no }\end{array}$ & Task no & $\begin{array}{l}\text { Min } \\
\text { node } \\
\text { no }\left(\mathrm{p}_{1}\right)\end{array}$ \\
\hline $\mathrm{fd} 4$ & $\begin{array}{l}\text { Detection of human faces, every } \\
4^{\text {th }} \text { frame, saving face image to } \\
\text { a file. }\end{array}$ & 1 & 1 & 4 & 1 \\
\hline fdt & $\begin{array}{l}\text { Detection of human faces using } \\
\text { data decomposition to } 32 \text { sub- } \\
\text { tasks. }\end{array}$ & 1 & 1 & 35 & 4 \\
\hline $\mathrm{fd} 32$ & $\begin{array}{l}\text { Detection of human faces every } \\
32^{\text {nd }} \text { frame, and notification } \\
\text { generation. }\end{array}$ & 1 & 1 & 5 & 1 \\
\hline $\mathrm{mrg}$ & $\begin{array}{l}\text { Merging of two streams and } \\
\text { providing time mark in the } \\
\text { output stream. }\end{array}$ & 2 & 1 & 3 & 1 \\
\hline
\end{tabular}

Two algorithms were to be under examination: MBFD, the proposed solution to providing accurate computation resources, with the correction related to the non-linear processor utilization increase, as well as its simplified version: BFD, which simply adds the task loads without the correction. The hypothesis was the former provides better scalability than the latter, thus its usage is justified for parallel, real-time multimedia stream processing.

During the test execution the consecutive benchmark services were started with an increasing number of compute nodes in the cluster. The minimum node 
number $p_{l}(s)$ depends on the service, and is directly related to the fixed reference productivity $l\left(p_{l}\right)$ used during scalability metric computation (see Eq. 5). Every experiment was repeated 5 times and the average value was considered. The measurements were continued until the maximum possible number of cluster nodes were used, in our case we could use 100 nodes at most, so $P_{M A X}=100$. Then the next service was tested, and after all tests and allocation algorithms are measured the procedure was finished. The experiments were performed for the services processing PAL video streams.

Figures 3 and 4 present the resulting scalability metric measured during the execution of the test services. The first chart consists of the results related to the BFD algorithm, the one based solely on the bin packing problem heuristic, and the second is related to

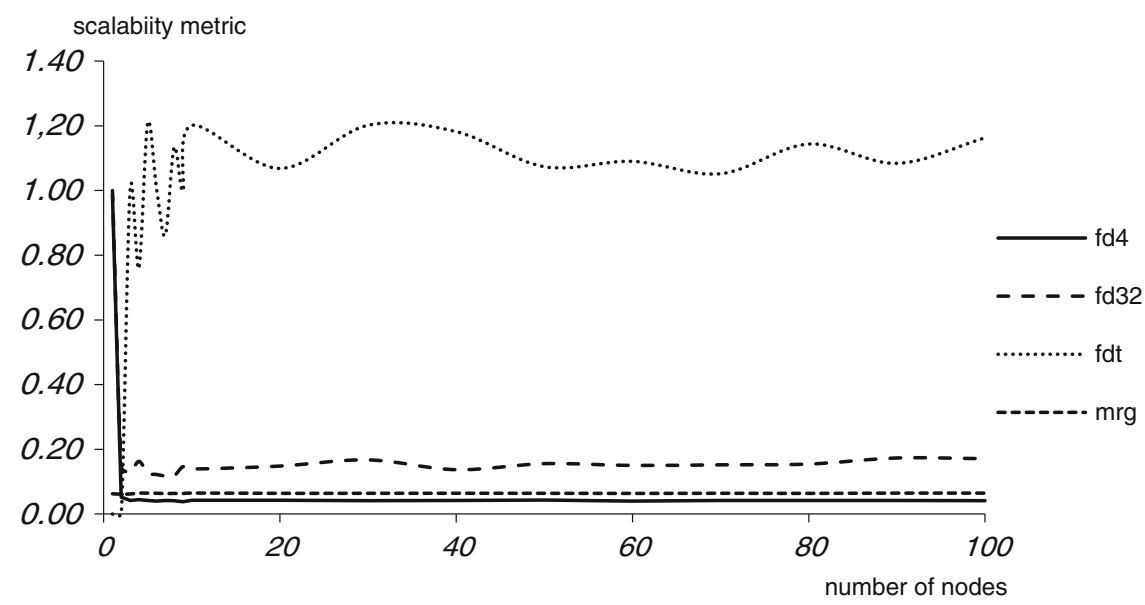

Fig. 3. The scalability metric measures for the BFD allocation algorithm

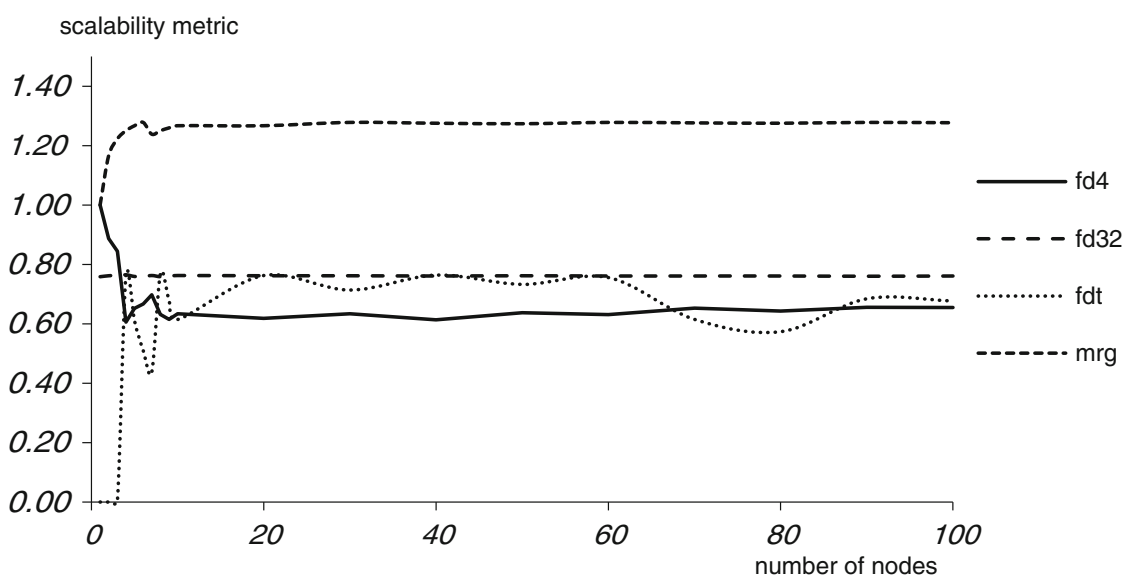

Fig. 4. The scalability metric measures for the MBFD allocation algorithm 
the MBFD algorithm, supported with the non-linear processor utilization prediction function. In general the smaller services (e.g. mrg with MBFD) have better scalability, however in other tests we observed the dependence of scalability value on the algorithms used in the services rather than the service complexity.

We can observe that in most cases the MBFD heuristic provides better results (higher scalability values), which is consistent with the assumption of non-linear load increase of the executed tasks. The only exception is related to the fdt service (which dropped down for MBFD), where the distribution of stream data to 32 tasks causes disappearance of the non-linear load increase effect - in this case, they behave like normal compute tasks. It is worth to mention the fluctuation of measurement for the larger services (consisting of a higher number of simple services, e.g. fdt), which is especially visible for a smaller number of nodes (but not only, see fdt for 60 + nodes using MBFD). It is caused by the service complexity, because quite often allocating an additional node does not increase the number of services executed in the cluster, e.g. for 4 and 5 nodes only one fdt service can be executed.

\section{Conclusions and Future Works}

In the article we proposed a new solution for stream processing task allocation in an HPC environment. The proposed heuristic is based on the observation of non-linear load increase for real-time streaming tasks and is related to the well-known BFD algorithm. The experimental results confirmed our first assumptions, providing the basis for the implementation of the allocation component for KASKADA platform [4].

In the future works, we are considering migration of the whole KASKADA platform [4] to the cloud environment, where the harder constraints related to more loose SLA in comparison to a typical HPC cluster can affect the performance of the allocation algorithm. On the other hand, we want to adapt the above solution to a heterogeneous environment, especially with the consideration of GPGPU devices.

\section{References}

1. Brandenburg, B.B., Calandrino, J.M., Anderson, J.H.: On the scalability of real-time scheduling algorithms on multicore platforms: a case study. In: Real-Time Systems Symposium, pp.157-169, 30 November-3 December (2008)

2. Gorlatch, S., Glinka, F., Ploss, A.: Towards a scalable real-time cyberinfrastructure for online computer games. In: 2009 15th International Conference on Parallel and Distributed Systems (ICPADS), pp.722-727, 8-11 December 2009

3. Jogalekar, P., Woodside, M.: Evaluating the scalability of distributed systems. IEEE Trans. Parallel Distrib. Syst. 11(6), 589-603 (2000)

4. Krawczyk, H., Proficz, J.: KASKADA - multimedia processing platform architecture. In: Proceedings of the 2010 International Conference on Signal Processing and Multimedia Applications (SIGMAP), pp.26-31, 26-28 July 2010

5. Kuck, D.J.: High Performance Computing, Challenges for Future Systems. Oxford University Press, New York (1996) 
6. Tang, Y., Changqin, B., Zhenghua, W., Sheng, S.: A scalable approach to distributed scheduling scheme on cluster architecture of real-time server. In: 2010 2nd International Conference on Computer Engineering and Technology (ICCET), vol. 3, pp. V3-123-V3-127, 16-18 April 2010

7. Krawczyk, H., Proficz, J., Daca, B.: Prediction of Processor Utilization for Real-Time Multimedia Stream Processing Tasks, Distributed Computing and Internet Technology, pp. 278-289. Springer, Heidelberg (2013)

8. Krawczyk, H., Proficz, J.: The task graph assignment for KASKADA platform. In: Proceedings of the 2010 International Conference on Software and Data Technologies (ICSOFT), pp. 192-197, 22-24 July 2010 